\title{
Joint Demosaicing and Super-Resolution Imaging from a Set of Unregistered Aliased Images
}

\author{
Patrick Vandewalle ${ }^{a}$, Karim Krichane ${ }^{a}$, David Alleysson $^{b}$, and Sabine Süsstrunk ${ }^{a}$ \\ ${ }^{a}$ School of Computer and Communication Sciences, Ecole Polytechnique Fédérale de Lausanne \\ (EPFL), CH-1015 Lausanne, Switzerland; \\ ${ }^{b}$ Laboratoire de Psychologie et Neurocognition, Université Pierre-Mendes France (UPMF), \\ F-38041 Grenoble, France.
}

\begin{abstract}
We present a new algorithm that performs demosaicing and super-resolution jointly from a set of raw images sampled with a color filter array. Such a combined approach allows us to compute the alignment parameters between the images on the raw camera data before interpolation artifacts are introduced. After image registration, a high resolution color image is reconstructed at once using the full set of images. For this, we use normalized convolution, an image interpolation method from a nonuniform set of samples. Our algorithm is tested and compared to other approaches in simulations and practical experiments.
\end{abstract}

Keywords: Demosaicing, super-resolution, image registration, aliasing.

\section{INTRODUCTION}

The resolution of an image taken with a digital camera is mainly determined by its lens and its sensor. If the modulation transfer function (MTF) of the lens removes too much of the high frequency scene information, the image will be blurred and details cannot be distinguished. Similarly, if the sampling frequency at the sensor (determined by the number of pixels and the sensor size) is lower than twice the maximum signal frequency passing through the optical system, the sampled scene is aliased. Details in the scene are not visible in the image because frequencies above half the sampling frequency are mapped into other frequencies below this limit. In both cases, the resolution or resolving power of the image is low.

Super-resolution algorithms combine the information present in multiple images of the same scene to reconstruct a high resolution image from a set of low resolution images. Typically, they use a set of aliased images that are captured by a single camera from slightly different positions. The images are first aligned, and then combined to construct a higher resolution image. This idea was first introduced by Tsai and Huang in $1984 .{ }^{1}$ Over the past twenty years, many approaches have been presented to solve this problem. A good overview of the current state of the art is given in the recent special issues of IEEE Signal Processing Magazine ${ }^{2}$ and EURASIP Journal on Applied Signal Processing. ${ }^{3}$

Most super-resolution algorithms can be decomposed into two parts: an image registration part followed by a reconstruction part. Very high accuracy is required in the registration (up to subpixel level) to be able to correctly reconstruct the high resolution image. Frequency domain algorithms typically estimate the linear phase difference between the Fourier transforms of the images. ${ }^{1,4}$ The motion is restricted to planar motion (shift and rotation), as this type of motion can be well described in frequency domain. Such algorithms can also account for aliasing directly in the Fourier domain description. ${ }^{5,6}$ Spatial domain methods use a Taylor series approximation to estimate the motion parameters, ${ }^{7}$ or compute salient features in the images and estimate the motion by mapping the features between the images. ${ }^{8}$ Spatial domain methods can be used for more complex

Further author information: (Send correspondence to Patrick Vandewalle) E-mail: \{Patrick.Vandewalle, Karim.Krichane, Sabine.Susstrunk\}@epfl.ch, David.Alleysson@upmf-grenoble.fr. This paper is reproducible. The code and data to reproduce the presented results, as well as some additional images are available online at http://lcavwww.epfl.ch/reproducible_research. 
motion models (projective transformations, etc.). They are also better adapted to estimate multiple motions in a single image, but it is more difficult to take aliasing into account.

Once the images are registered, a robust reconstruction method is needed to build a high resolution image from the set of irregularly spaced samples (pixels) and undo the blur caused by the optical system. An overview of existing reconstruction methods is given by Park et al. ${ }^{9}$ Tsai and Huang ${ }^{1}$ presented a frequency domain approach to compute the high resolution Fourier coefficients from the aliased images. Most other reconstruction methods are applied in the spatial domain, and use nonuniform interpolation, ${ }^{10,11}$ iterative back projections, ${ }^{7,12}$ or maximum a posteriori and maximum likelihood methods ${ }^{8,13}$ to compute a high resolution image from the set of aligned low resolution input images. Some bounds on the performance of super-resolution algorithms have been recently presented by Robinson and Milanfar ${ }^{14}$ and Baker and Kanade. ${ }^{15}$

Typically, such super-resolution algorithms are applied on images captured with a digital camera. Most digital cameras use a single sensor with a color filter array (CFA). At each pixel position, the sensor measures either the red, green, or blue value of the image. A demosaicing algorithm is then applied to compute the full color image from this CFA image. This can be a simple bilinear interpolation or a more complex algorithm using for example the correlation between the different color channels. ${ }^{16}$ Alleysson et al. presented a new method that uses the separation of the luminance and chrominance information in the Fourier spectrum of the Bayer CFA image. ${ }^{17}$ We will use this approach to extract the high resolution luminance information from the images.

The separate application of a demosaicing and a super-resolution algorithm is sub-optimal, as artifacts introduced by the demosaicing (such as color aliasing) will be considered as part of the signal in the subsequent super-resolution algorithm. Aliasing artifacts introduced in the demosaicing process can therefore not be removed anymore in the super-resolution algorithm. This will result in a lower performance of the reconstruction algorithm, and to a minor extent also the registration algorithm. In this paper, we will therefore present an algorithm for joint demosaicing and super-resolution. We first take advantage of the separation of luminance and chrominance in the Fourier transform of the Bayer CFA images to perform a precise frequency domain image registration. Then, we split the Bayer CFA images into luminance and chrominance. Next, we separately interpolate the high resolution luminance and chrominance information using the information from all the input images. Finally, we combine the high resolution luminance and chrominance images again to construct a high resolution image with less color aliasing artifacts. A similar approach was presented by Farsiu et al. ${ }^{18}$ They presented a maximum a posteriori approach for demosaicing and super-resolution reconstruction from a set of previously aligned input images. In their mathematical model, they include separate penalty terms for the raw data fidelity, the sharpness of the luminance information, the smoothness of the chrominance information, and the intercolor homogeneity of edge location and orientation.

Our joint demosaicing and super-resolution approach will be presented in Section 2] The results using our algorithm will be compared to other approaches in Section 3 Section 4 concludes the article.

\section{ALGORITHM}

Our algorithm is based on the idea presented by Alleysson et al. ${ }^{17}$ that luminance and chrominance information are encoded separately in the Fourier spectrum of a Bayer CFA image. They showed that a Bayer CFA image $I_{C F A}(x, y)$ can be written as a sum of the red, green, and blue color channels:

$$
I_{C F A}(x, y)=\sum_{i=1}^{3} C_{i}(x, y) M_{i}(x, y) \quad \text { with } \begin{cases}M_{1}(x, y)=(1+\cos (\pi x))(1+\cos (\pi y)) / 4 & \text { (red) } \\ M_{2}(x, y)=(1-\cos (\pi x) \cos (\pi y)) / 2 & \text { (green) } \\ M_{3}(x, y)=(1-\cos (\pi x))(1-\cos (\pi y)) / 4 & \text { (blue) }\end{cases}
$$

The image $C_{i}(x, y)$ is the $i$-th color channel image, and $M_{i}(x, y)$ is a modulation matrix, which is 1 only at the measured positions of the image, and 0 elsewhere. As these modulation functions are a combination of cosines, their Fourier transform is a combination of Diracs. We define $r$ and $s$ as the image width and height, respectively. 
Using the fact that a product in spatial domain corresponds to a convolution in frequency domain, we obtain

$$
\begin{aligned}
\mathbf{I}_{C F A}(k, l)= & \frac{1}{2}\left(\mathbf{C}_{1}(k, l)+2 \mathbf{C}_{2}(k, l)+\mathbf{C}_{3}(k, l)\right) \\
& +\frac{1}{8}\left(\mathbf{C}_{1}(k, l)-\mathbf{C}_{3}(k, l)\right) *\left(\delta\left(k-\frac{r}{2}\right) \delta(l)+\delta\left(k+\frac{r}{2}\right) \delta(l)+\delta(k) \delta\left(l-\frac{s}{2}\right)+\delta(k) \delta\left(l+\frac{s}{2}\right)\right) \\
& +\frac{1}{16}\left(\mathbf{C}_{1}(k, l)-2 \mathbf{C}_{2}(k, l)+\mathbf{C}_{3}(k, l)\right) \\
& \quad *\left(\delta\left(k-\frac{r}{2}\right) \delta\left(l-\frac{s}{2}\right)+\delta\left(k+\frac{r}{2}\right) \delta\left(l-\frac{s}{2}\right)+\delta\left(k-\frac{r}{2}\right) \delta\left(l+\frac{s}{2}\right)+\delta\left(k+\frac{r}{2}\right) \delta\left(l+\frac{s}{2}\right)\right),
\end{aligned}
$$

where Fourier transforms are indicated in bold.

At the same time, we can also write a color image $I(x, y)$ as a sum of a scalar representing its luminance $\Phi(x, y)$ and a length three vector $\Psi(x, y)$ that is called chrominance and represents opponent colors:

$$
I(x, y)=\left(\begin{array}{l}
C_{1}(x, y) \\
C_{2}(x, y) \\
C_{3}(x, y)
\end{array}\right)=\Phi(x, y)+\left(\begin{array}{c}
\Psi_{1}(x, y) \\
\Psi_{2}(x, y) \\
\Psi_{3}(x, y)
\end{array}\right) .
$$

If we define the luminance as $\Phi=\left(C_{1}+2 C_{2}+C_{3}\right) / 2$, we obtain

$$
I(x, y)=\left(\begin{array}{l}
C_{1}(x, y) \\
C_{2}(x, y) \\
C_{3}(x, y)
\end{array}\right)=\left(C_{1}(x, y)+2 C_{2}(x, y)+C_{3}(x, y)\right) / 2+\frac{1}{2}\left(\begin{array}{c}
-2 C_{2}(x, y)-C_{3}(x, y) \\
-C_{1}(x, y)-C_{2}(x, y)-C_{3}(x, y) \\
-C_{1}(x, y)-2 C_{2}(x, y)
\end{array}\right) .
$$

Using this definition, we can also see that the first term in (2) corresponds to the luminance signal $\Phi(x, y)$, and hence the two other terms represent the chrominance $\Psi(x, y)$. Due to the modulation functions, the chrominance parts are represented in the high frequency parts of the spectrum, and are therefore separated from the luminance, which is represented in the low frequencies. A visual illustration is given in Figure 1(a)

This separate encoding can be used both for the image registration and the reconstruction. Image registration is typically performed on grayscale images, and should therefore be applied only to the luminance part of the images. Using a lowpass filter, we can extract the luminance information from the images, and use these to estimate the registration parameters. In this paper, we will use a frequency domain approach that uses only the low frequencies for image registration, which are less prone to aliasing. As this is also the part of the CFA Fourier transform that contains the luminance information, we can apply our algorithm directly on the raw CFA images. Next, we separate the images into luminance and chrominance using a lowpass filter, and interpolate the two separately.

\subsection{Overview}

Our algorithm consists of the following main steps. A block diagram is given in Figure 2

1. Image Registration: Align the set of images pairwise using the low frequency (luminance) information of the CFA Fourier transform images.

2. Luminance/Chrominance Separation: Extract the luminance and chrominance information from each of the input images.

3. Image Reconstruction: Interpolate a high resolution image using the data from the set of images. We used a normalized convolution method for the interpolation. The luminance and chrominance information are interpolated separately and combined afterwards. 


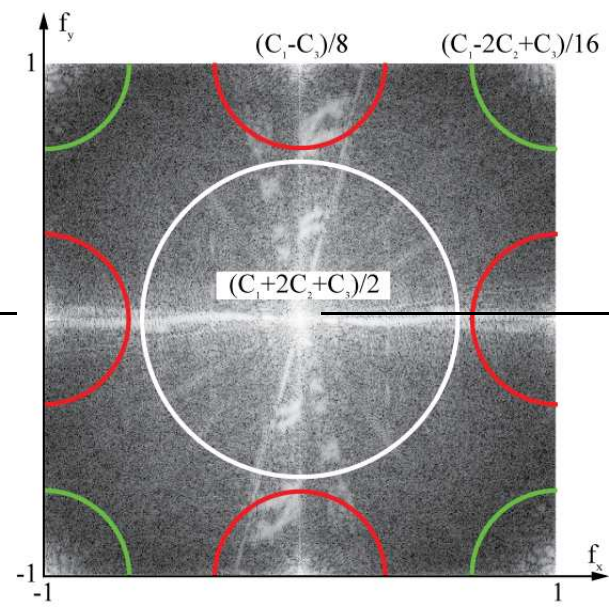

(a)

$$
F=\left(\begin{array}{rrrrrrrrrrr}
0 & 0 & 0 & 0 & 1 & 0 & 1 & 0 & 0 & 0 & 0 \\
0 & 0 & 0 & -1 & 0 & -2 & 0 & -1 & 0 & 0 & 0 \\
0 & 0 & 1 & 1 & 2 & 1 & 2 & 1 & 1 & 0 & 0 \\
0 & -1 & 1 & -5 & 3 & -9 & 3 & -5 & 1 & -1 & 0 \\
1 & 0 & 2 & 3 & 1 & 7 & 1 & 3 & 2 & 0 & 1 \\
0 & -2 & 1 & -9 & 7 & 104 & 7 & -9 & 1 & -2 & 0 \\
1 & 0 & 2 & 3 & 1 & 7 & 1 & 3 & 2 & 0 & 1 \\
0 & -1 & 1 & -5 & 3 & -9 & 3 & -5 & 1 & -1 & 0 \\
0 & 0 & 1 & 1 & 2 & 1 & 2 & 1 & 1 & 0 & 0 \\
0 & 0 & 0 & -1 & 0 & -2 & 0 & -1 & 0 & 0 & 0 \\
0 & 0 & 0 & 0 & 1 & 0 & 1 & 0 & 0 & 0 & 0
\end{array}\right) / 128
$$

(b)

Figure 1. Separate luminance/chrominance encoding. (a) Fourier transform of the CFA image showing the encoding of luminance and chrominance at different locations. (b) Filter $F$ used to extract the luminance information.

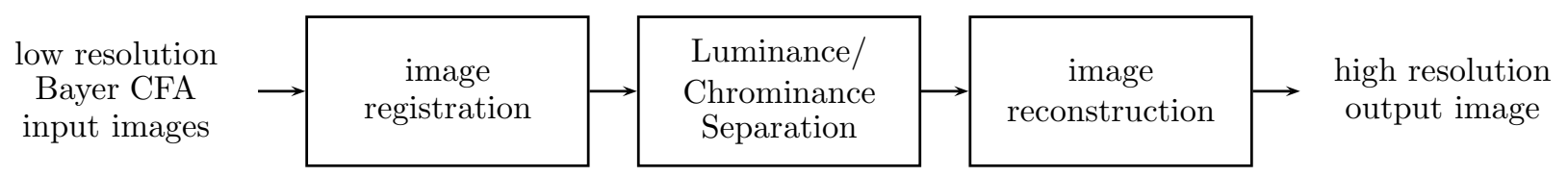

Figure 2. Block diagram of the proposed algorithm.

\subsection{Image Registration}

First of all, the Bayer CFA input images need to be precisely aligned. We use the frequency domain approach presented by Vandewalle et al. ${ }^{4}$ This algorithm selects only the low frequency information because this part of the spectrum is less corrupted by aliasing. In our case, this is also the part of the spectrum encoding the luminance information (see (2)). We can therefore directly apply our registration algorithm to the raw CFA images. We first perform a planar rotation estimation, followed by a planar shift estimation. The rotation angle is estimated by computing the frequency content $H(\alpha)$ of the image as a function of the angle for each of the input images:

$$
H(\alpha)=\int_{\alpha-\Delta \alpha / 2}^{\alpha+\Delta \alpha / 2} \int_{0}^{\infty}\left|\mathbf{I}_{C F A}(r, \theta)\right| d r d \theta
$$

where $\mathbf{I}_{C F A}(r, \theta)$ is the Fourier transform of the CFA image $I_{C F A}$, converted in polar coordinates. The rotation angle between two images can then be found at the maximum of the correlation between two such functions. Next, the rotation is canceled, and the shifts are estimated by computing the least squares fit of a plane through the (linear) phase difference between the images.

As we only use the low frequency information of the images, we do not need to separate luminance and chrominance for this phase. The use of the raw sensor data for the image alignment allows a higher precision of the alignments, as no additional filtering or interpolation errors are introduced.

\subsection{Luminance/Chrominance Separation}

Next, we separate the luminance and chrominance information in each of the images in order to interpolate them separately. As indicated in (2), we can extract the luminance signal from the CFA images using a low-pass filter. We use the filter $F$ specified by Alleysson et al. ${ }^{17}$ (see Figure 1(b)]. The three chrominance parts (for red, green and blue) are then obtained by subtracting this luminance information from the red, green and blue channels of 
the CFA image and demodulating the result. This results in a luminance image $\Phi$ and three chrominance images $\Psi_{1}, \Psi_{2}$, and $\Psi_{3}$, all at the original image size:

$$
\begin{aligned}
\Phi & =I_{C F A} * F \\
\Psi_{1} & =\left(\left(I_{C F A}-\Phi\right) \odot M_{1}\right) * D_{1} \\
\Psi_{2} & =\left(\left(I_{C F A}-\Phi\right) \odot M_{2}\right) * D_{2} \\
\Psi_{3} & =\left(\left(I_{C F A}-\Phi\right) \odot M_{3}\right) * D_{1} \\
& \text { with } D_{1}=\left(\begin{array}{lll}
1 & 2 & 1 \\
2 & 4 & 2 \\
1 & 2 & 1
\end{array}\right) / 4, \quad D_{2}=\left(\begin{array}{lll}
0 & 1 & 0 \\
1 & 4 & 1 \\
0 & 1 & 0
\end{array}\right) / 4 .
\end{aligned}
$$

The matrices $D_{1}$ and $D_{2}$ are two demodulation (or interpolation) filters, and the symbol $\odot$ is used for a pointwise multiplication of two matrices.

\subsection{Image Reconstruction}

Now that the luminance and chrominance signals are separated (and demodulated), we can compute their high resolution versions. We apply the normalized convolution approach by Pham et al. ${ }^{11}$ on each of the four channels separately. They adapted normalized convolution, ${ }^{19}$ a technique for local signal modeling from projections onto a basis, for image interpolation from a nonuniform set of samples. Pixel values of the high resolution image are computed by fitting a polynomial surface (linear in our case) through the samples in a neighborhood around the pixel. A Gaussian weighting function is used (called applicability function) to have the highest contributions from samples close to the considered pixel. In our implementation we used a variance $\sigma^{2}=2$. A pixel of the high resolution image is computed from the pixels in a neighborhood around it as

$$
p=\left(B^{T} W B\right)^{-1} B^{T} W f,
$$

where $f$ is an $N \times 1$ vector containing the neighborhood pixels, $B$ is an $N \times m$ matrix of $m$ basis functions sampled at the local coordinates of the pixels $f$, and $W$ is an $N \times N$ weighting matrix containing the Gaussian weights sampled at the pixel coordinates. The first element of the $m \times 1$ vector $p$ gives the interpolated pixel value. For our neighborhood, we used a circular region with radius four times the pixel distance of the high resolution image. Due to the nonuniform grid, the number of pixels $N$ in this region may vary depending on the position.

We perform this interpolation for the luminance channel $\Phi$, as well as for each of the chrominance channels $\Psi_{1}, \Psi_{2}$, and $\Psi_{3}$. After the reconstruction, the luminance and chrominance are added together again, which results in the final high resolution color image.

\section{RESULTS}

We have tested our algorithm in a number of simulations and practical experiments, and compared its performance to some other approaches. The simulations allowed us to test the different parts of our algorithm separately, and to compare the results to a ground truth. The experiments allowed us then to test our approach on some real images.

\subsection{Compared Methods}

First of all, we compared our combined super-resolution-demosaicing approach to a single image that was demosaiced using the algorithm by Alleysson et al. ${ }^{17}$ As input for the demosaicing algorithm, we used one image from the set of images used for the super-resolution. This gives an indication about the resolution that is gained by our super-resolution algorithm. Typically, if the image alignment is performed poorly, the results using a single image would be better.

Next, we compared it to a standard super-resolution approach, i.e. demosaicing the input images first (using the algorithm by Alleysson et al.), and then applying super-resolution to the resulting images. Finally, 
we compared the results using our algorithm to the results using the super-resolution-demosaicing algorithm by Farsiu et al., ${ }^{18}$ which is to our knowledge the only other algorithm performing joint demosaicing and superresolution. For this comparison, we used the implementation by the authors (MDSP super-resolution software ${ }^{20}$ ). In this algorithm, there are a number of parameters that have to be optimized manually. We did this optimization as far as we could, but cannot guarantee that these are the best parameters possible.

\subsection{Simulations}

In our simulations, we generated CFA images by shifting the image first, and then subsampling to obtain the CFA image. We first tested the performance of the image registration algorithm in 250 simulations with random shifts (Gaussian distribution with $\sigma=2$ ) and rotations (Gaussian distribution with $\sigma=0.5$ ), and compared the results with those using the demosaiced images. The average absolute errors are given in Table 1 As could be expected, the highest performance is obtained by applying the registration algorithm directly on the full CFA images. However, the results are very similar, which can be explained by the fact that both algorithms use the low frequency information, which is typically not changed very much in demosaicing.

Table 1. Image registration performance. Our algorithm (CFA registration) is compared to registration of the demosaiced images. Average absolute errors are given for shift (pixels) and rotation (degrees).

\begin{tabular}{lcc}
\hline & CFA registration & demosaiced registration \\
\hline shift & 0.1892 & 0.1912 \\
rotation & 0.5297 & 0.5371 \\
\hline
\end{tabular}

Next, we tested the super-resolution and demosaicing algorithm for known motion parameters. For this simulation, we shifted the images first, downsampled them by two, and subsampled them to create CFA images. The results can be seen in Figure 3and 4 *, where they are compared to the single demosaiced image, the separate demosaicing and super-resolution, and the result obtained using the algorithm by Farsiu et al. We clearly see less color aliasing than with the single demosaiced image. Our results are essentially the same as those with the separate processing, which can be explained by the small amount of color aliasing and the idealized setup. The shifts used for this simulation are exactly 0.5 pixels, so that the information is uniformly available over the image surface when the images are combined. The results with the algorithm by Farsiu et al. (as we obtained them using the MDSP software ${ }^{20}$ ) show more color aliasing artifacts. Our images are also less sharp than the results using Farsiu et al.'s algorithm, which is mainly due to a sub-optimal luminance/chrominance separation in our algorithm. This can be solved by further optimizing the luminance selection filter, ${ }^{17,21}$ or using an additional sharpening step.

\subsection{Experiments}

For the first practical experiment, we took a set of four pictures of a resolution chart using a Canon EOS 350D digital camera. The pictures were stored in raw format, such that we had access to the raw Bayer CFA data. First, we selected a part of these 8 megapixel images to reduce computation times and memory requirements. These parts were then processed using the different algorithms, and the results are shown in Figure 5

The second experiment was similar to the first one, but now we took a set of four aliased images using a Logitech QuickCam Fusion webcam. These images were also captured in raw Bayer CFA format, and then processed using the above algorithms. Figure 6 shows the results of this experiment.

In both experiments we have less color aliasing with the presented method than with demosaicing of a single image, and we can correctly distinguish higher frequency patterns in the resulting image. However, the results

\footnotetext{
${ }^{*}$ Note that the results shown in this paper might contain artifacts due to image compression in the pdf generation and printer resolution. A full resolution, uncompressed version of the images is available online at http://lcavwww.epfl.ch/reproducible_research.
} 
of the separate and combined algorithms are again very similar. As already discussed, the precision of the image registration is very similar in the two approaches. For the reconstruction, both are based on a lowpass filter to separate luminance and chrominance. The results can be explained by the fact that most of the artifacts come from an inaccurate separation in both approaches. As in the simulations, the results using the algorithm by Farsiu et al. contain more color aliasing artifacts but are also sharper than with the other approaches.

\section{CONCLUSIONS}

We have presented a new algorithm to perform joint demosaicing and super-resolution from a set of Bayer CFA input images of a scene. Between the input images, there is some small, unknown motion that can be modeled as planar motion. First, we estimate the motion using a frequency domain method on the low frequency information of the Bayer CFA images. Next, we separate luminance and chrominance for each of the input images, and compute the high resolution luminance and chrominance images separately. Finally, they are combined to form a high resolution color image. Simulations and practical experiments show that this joint approach has good results. The results obtained with this algorithm are very similar to those obtained using a separate demosaicing and super-resolution setup. This can be explained by the fact that the different processing steps in both approaches are very similar. The main source of errors is probably the separation between luminance and chrominance. If this separation is not accurately done, parts of the luminance signal remain in the chrominance signals, and will cause artifacts. In future work, we will therefore look further into an optimization of these separation filters. ${ }^{17,21}$ We also compared our approach to the combined algorithm by Farsiu et al., ${ }^{18}$ where we used parameter values close to the default values.

\section{ACKNOWLEDGMENTS}

The authors would like to thank Remy Zimmermann and his team at Logitech for the use of their cameras and tools to extract raw Bayer CFA data.

\section{REFERENCES}

1. R. Y. Tsai and T. S. Huang, "Multiframe image restoration and registration," in Advances in Computer Vision and Image Processing, T. S. Huang, ed., 1, pp. 317-339, JAI Press, 1984.

2. "IEEE Signal Processing Magazine, special issue on super-resolution," May 2003.

3. "EURASIP journal on applied signal processing, special issue on super-resolution," 2006.

4. P. Vandewalle, S. Süsstrunk, and M. Vetterli, "A Frequency Domain Approach to Registration of Aliased Images with Application to Super-Resolution," EURASIP Journal on Applied Signal Processing, Special Issue on Super-Resolution Imaging 2006, 2006. Article ID 71459, 14 pages.

5. P. Vandewalle, L. Sbaiz, J. Vandewalle, and M. Vetterli, "How to Take Advantage of Aliasing in Bandlimited Signals," in IEEE International Conference on Acoustics, Speech and Signal Processing, 3, pp. 948-951, May 2004.

6. P. Vandewalle, L. Sbaiz, M. Vetterli, and S. Süsstrunk, "Super-Resolution from Highly Undersampled Images," in IEEE International Conference on Image Processing, 1, pp. 889-892, Sept. 2005.

7. D. Keren, S. Peleg, and R. Brada, "Image sequence enhancement using sub-pixel displacement," in Proceedings IEEE Conference on Computer Vision and Pattern Recognition, pp. 742-746, June 1988.

8. D. Capel and A. Zisserman, "Computer vision applied to super resolution," IEEE Signal Processing Magazine 20, pp. 75-86, May 2003.

9. S. C. Park, M. K. Park, and M. G. Kang, "Super-resolution image reconstruction: A technical overview," IEEE Signal Processing Magazine 20, pp. 21-36, May 2003.

10. A. Papoulis, "Generalized sampling expansion," IEEE Transactions on Circuits and Systems 24, pp. 652654, Nov. 1977.

11. T. Q. Pham, L. J. van Vliet, and K. Schutte, "Robust Fusion of Irregularly Sampled Data Using Adaptive Normalized Convolution," EURASIP Journal on Applied Signal Processing 2006, 2006. Article ID 83268, 12 pages. 
12. S. Farsiu, D. Robinson, M. Elad, and P. Milanfar, "Advances and challenges in super-resolution," International Journal of Imaging Systems and Technology 14, pp. 47-57, Aug. 2004.

13. M. Elad and A. Feuer, "Restoration of a single superresolution image from several blurred, noisy, and undersampled measured images," IEEE Transactions on Image Processing 6, pp. 1646-1658, December 1997.

14. D. Robinson and P. Milanfar, "Statistical performance analysis of super-resolution," IEEE Transactions on Image Processing 15, pp. 1413-1428, June 2006.

15. S. Baker and T. Kanade, "Limits on super-resolution and how to break them," IEEE Transactions on Pattern Analysis and Machine Intelligence 24, pp. 1167-1183, Sept. 2002.

16. R. Kimmel, "Demosaicing: Image Reconstruction from Color CCD Samples," IEEE Transactions on Image Processing 8, pp. 1221-1228, Sept. 1999.

17. D. Alleysson, S. Süsstrunk, and J. Hérault, "Linear demosaicing inspired by the human visual system," IEEE Transactions on Image Processing 14(4), pp. 439-449, 2005.

18. S. Farsiu, M. Elad, and P. Milanfar, "Multi-Frame Demosaicing and Super-Resolution of Color Images," IEEE Transactions on Image Processing 15, pp. 141-159, Jan. 2006.

19. H. Knutsson and C.-F. Westin, "Normalized and Differential Convolution: Methods for Interpolation and Filtering of Incomplete and Uncertain Data," in Proc. IEEE Conference on Computer Vision and Pattern Recognition (CVPR), pp. 515-523, 1993.

20. S. Farsiu, D. Robinson, and P. Milanfar, "MDSP resolution enhancement software," 2004.

21. E. Dubois, "Filter Design for Adaptive Frequency-Domain Bayer Demosaicking," in Proc. IEEE International Conference on Image Processing, pp. 2705-2708, Oct. 2006. 


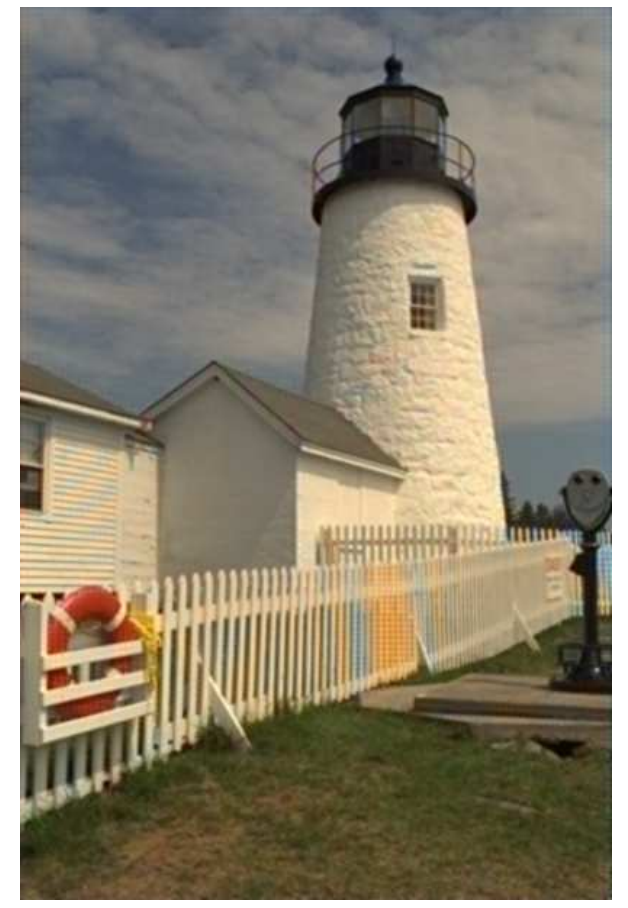

(a)

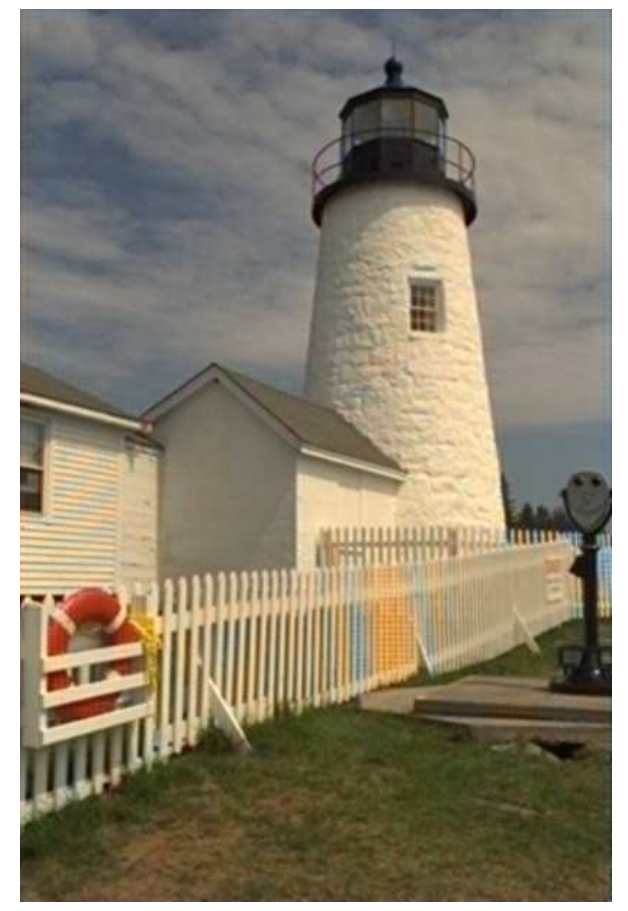

(c)

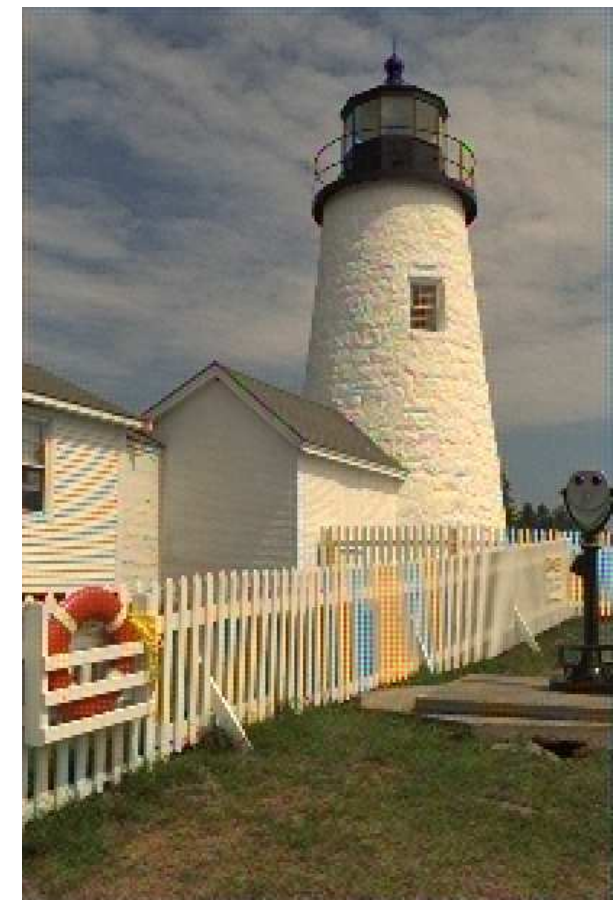

(b)

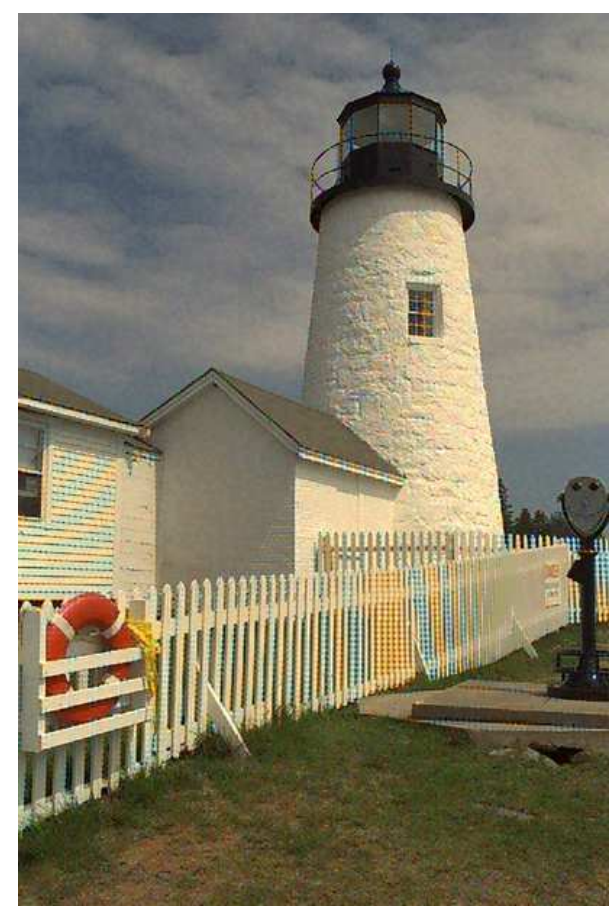

(d)

Figure 3. Simulation results. (a) Result using the presented algorithm. (b) Result using demosaicing on a single image. (c) Result using demosaicing followed by super-resolution. (d) Result using the algorithm by Farsiu et al. 


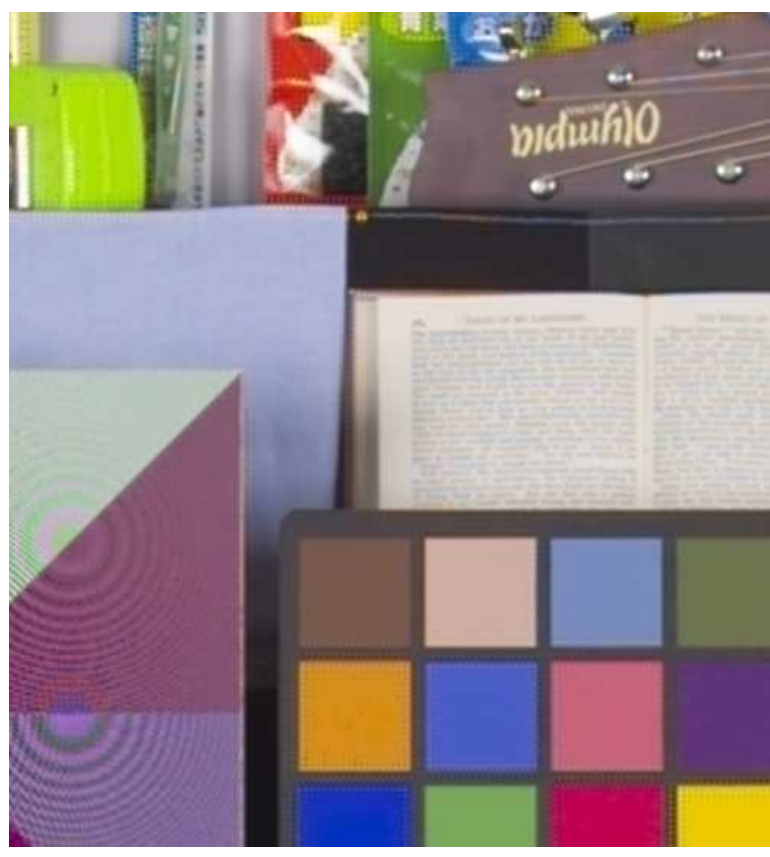

(a)

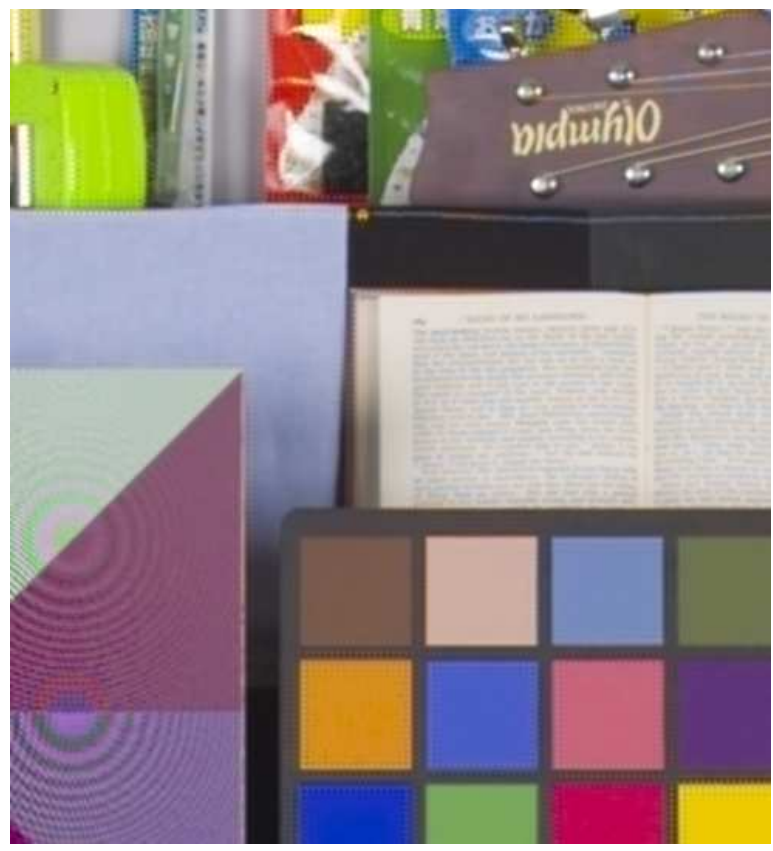

(c)

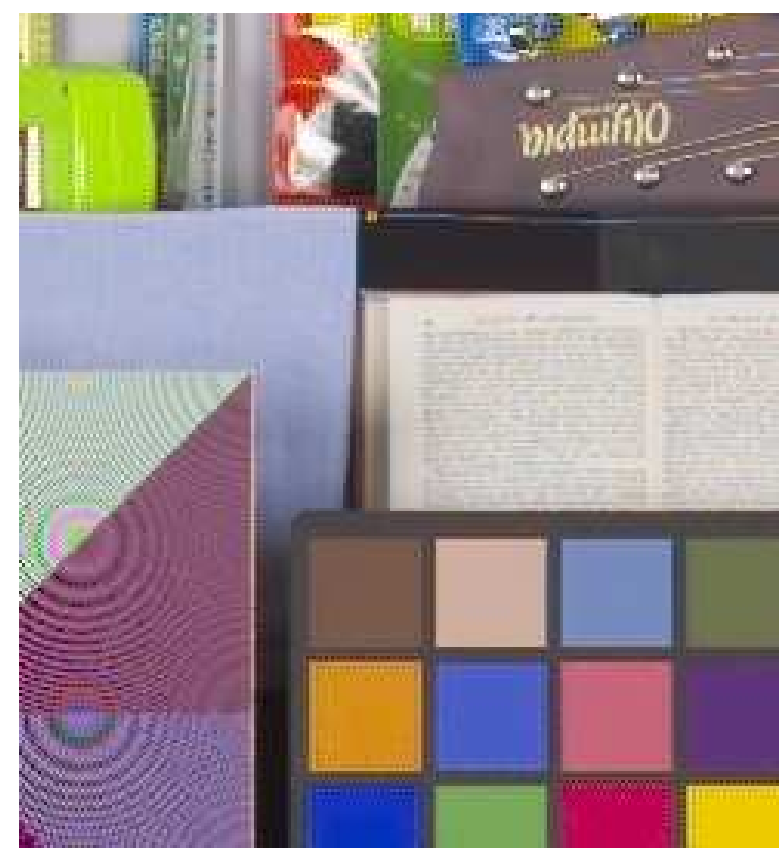

(b)

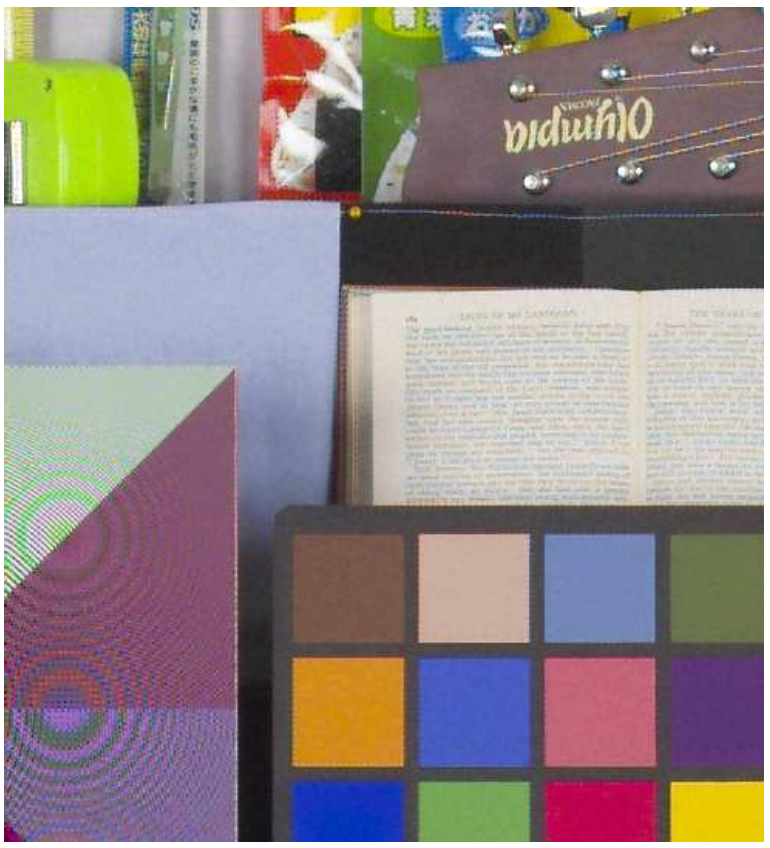

(d)

Figure 4. Simulation results. (a) Result using the presented algorithm. (b) Result using demosaicing on a single image. (c) Result using demosaicing followed by super-resolution. (d) Result using the algorithm by Farsiu et al. 


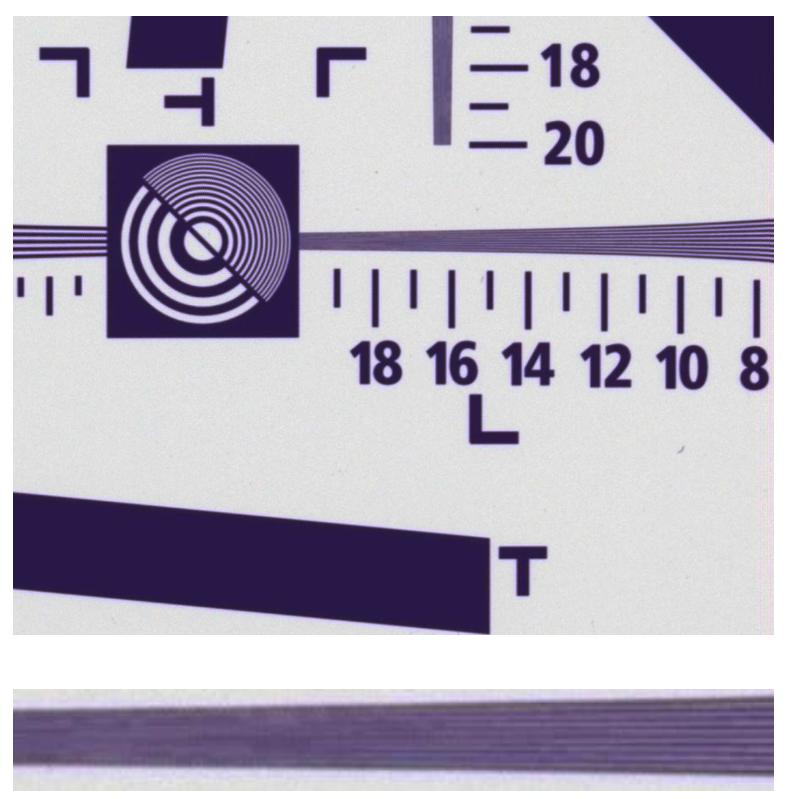

(a)

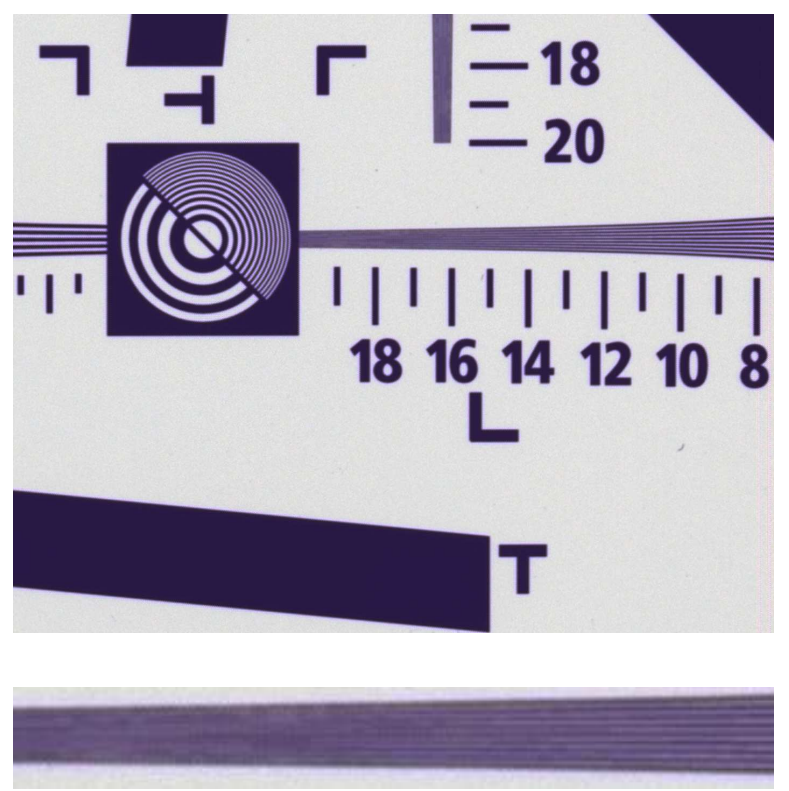

(c)
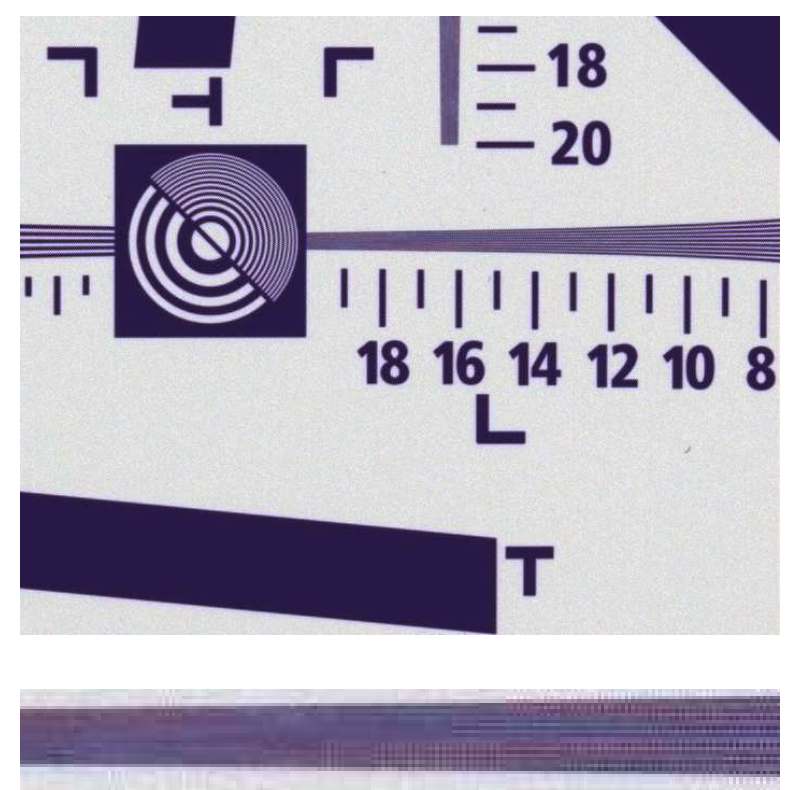

(b)

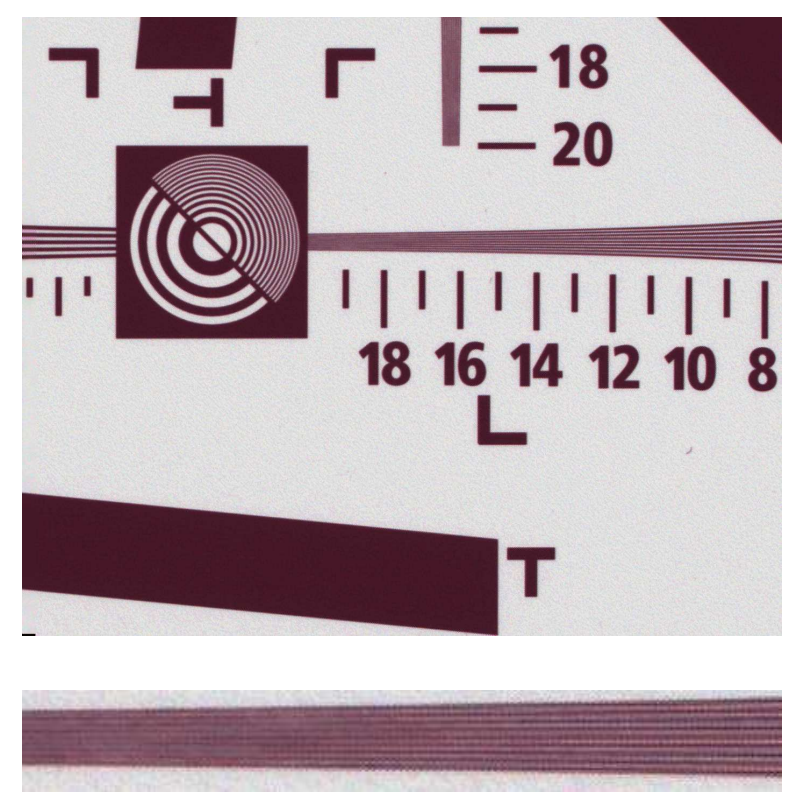

(d)

Figure 5. First experiment. (a) Result using the presented algorithm. (b) Result using demosaicing on a single image. (c) Result using demosaicing followed by super-resolution. (d) Result using the algorithm by Farsiu et al. 


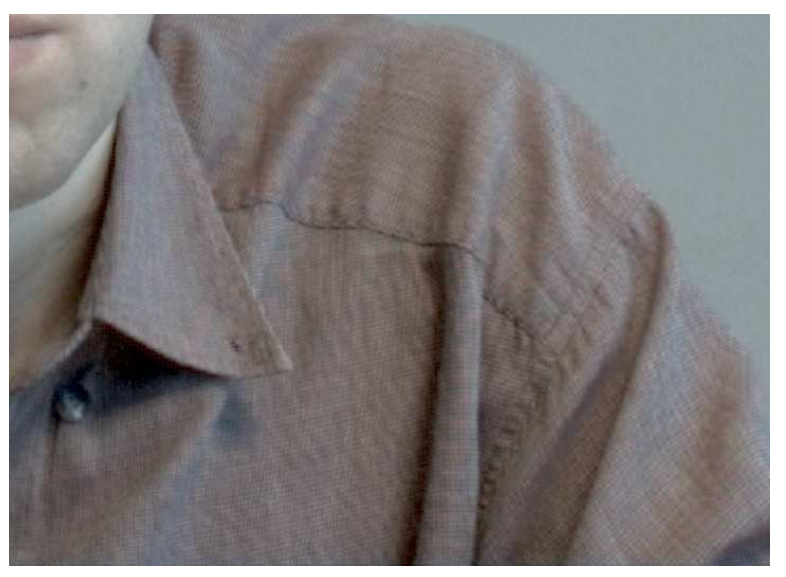

(a)

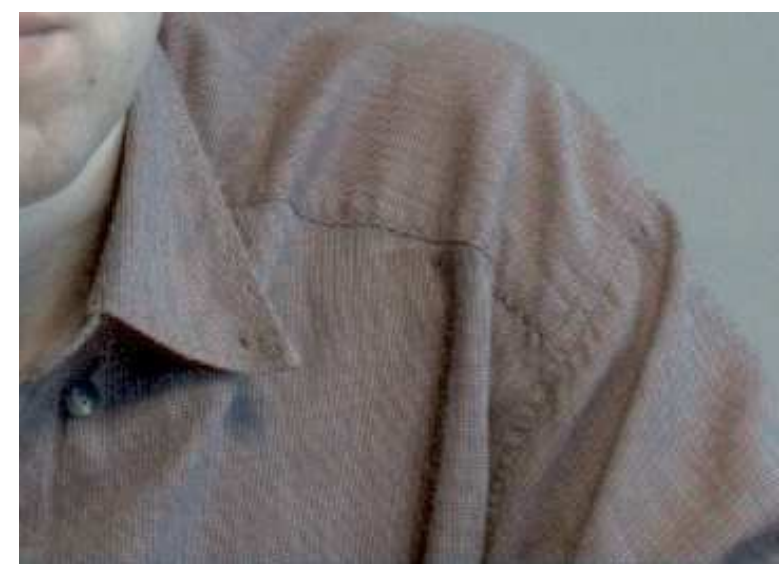

(b)

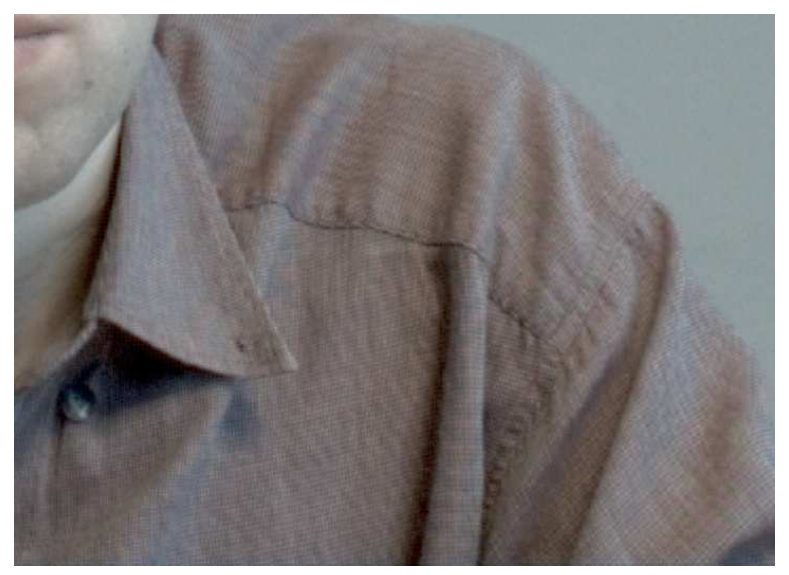

(c)

Figure 6. Second experiment. (a) Result using the presented algorithm. (b) Result using demosaicing on a single image. (c) Result using demosaicing followed by super-resolution. 